Conclusions: A new score was introduced with the aim to predict digital ulcers. If applied correctly and with the new imaging techniques proposed, $95 \%$ of patients at risk of digital ulcers throughout 12 months could be identified.

Disclosure of Interest: None declared

DOI: 10.1136/annrheumdis-2018-eular.6230

\section{AB0810 A COMPARISON OF CLINICAL PRESENTATION AND INCIDENCE RATE OF CARDIOPULMONARY INVOLVEMENT BETWEEN MALE AND FEMALE PATIENTS WITH EARLY SYSTEMIC SCLEROSIS}

S. Wangkaew ${ }^{1}$, S. Tungteerabunditkul ${ }^{1}$, V. Sawangduan ${ }^{1}$, N. Prasertwittakij ${ }^{2}$, J. Euathrongchit ${ }^{3} .{ }^{1}$ Rheumatology; ${ }^{2}$ Cardiology, ${ }^{3}$ Diagnostic Radiology, CHIANG MAI UNIVERSITY, Chiang Mai, Thailand

Background: Data regarding the incidence rate (IR) of cardiopulmonary involvement in comparison between male and female patients with early systemic sclerosis (SSc) are limited.

Objectives: To compare the prevalence of clinical manifestations and the IR of cardiopulmonary involvement comparing between male and female patients with early SSc.

Methods: An inception cohort of early SSc patients (disease duration $\leq 3$ years from the first non-Raynaud's phenomenon) seen at the Rheumatology clinic, Maharaj Nakorn Chiang Mai Hospital, Thailand, between January 2010 and June 2016, was used. All patients were assessed for clinical manifestations and underwent ECG, echocardiography, and HRCT at the study entry and every 12 months thereafter. In the last visit, the cumulative clinical manifestations were recorded. Patients who continue to follow up at least 12 months were included for analysis.

Results: One hundred and fifteen patients ( $89 \mathrm{dcSSc}$ ) with a mean (SD) disease duration of 11.6 (8.8) months at cohort entry were enrolled during a mean (SD) observation period of $3.8(1.6)$ years. There were 46 male patients $(40 \%)$. Tests for anti-topoisomerase I and anti-centromere antibodies were positive in 91 $(79.1 \%)$ and $7(6.1 \%)$ patients, respectively. The male group had higher prevalence of dcSSc subtype ( $91.3 \%$ vs. $69.5 \%, p=0.006)$ and positive test for anti-topoisomerase I antibody ( $89.1 \%$ vs. $72.5 \%, p=0.031)$ compared with the female group. At enrollment, the male group had higher prevalence of hypo-hyperpigmentation $(84.8 \%$ vs. $65.2 \%, p=0.021)$, suspected myositis $(26.1 \%$ vs. $10.1 \%$, $\mathrm{p}=0.024)$, and right ventricular dysfunction $(8.7 \%$ vs. $0 \%, p=0.024)$ compared with the female group. In the last visit, the male group had higher cumulative prevalence of digital ulcer $(47.8 \%$ vs. $27.5 \%, p=0.026)$, telangiectasia $(93.5 \%$ vs. $69.6 \%, p=0.002)$, joint contracture $(69.6 \%$ vs. $43.5 \%, p=0.006)$, tendon friction rub ( $39.1 \%$ vs. $20.3 \%, p=0.027)$, LVEF $<50 \%(21.7 \%$ vs. $8.7 \%, p=0.048)$ and right ventricular dysfunction ( $34.8 \%$ vs. $7.2 \%, \mathrm{p}<0.001$ ) compared with the female group. The male group had significant higher IR of right ventricular dysfunction (8.21 vs. 1.99 per 100 person-years, $\mathrm{p}=0.006$ ) and interstitial lung disease (ILD) determined by HRCT (63.66 vs. 39.19 per 100 person-years, $p=0.022$ ) compared to the female group. However, no significant IR of LVEF $<50 \%$ (5.63 vs. 2.10 per 100 personyears, $\mathrm{p}=0.078$ ), and elevated systolic pulmonary artery pressure $\geq 50 \mathrm{mmHg}$ determined by echocardiography ( 2.81 vs. 1.55 per 100 person-years, $p=0.339$ ) were observed among the two groups.

Conclusions: Our study cohort found that male gender had higher proportion of dcSSc subtype and had more severe clinical manifestations compared with female. In addition, during the follow-up period, the IR of right ventricular dysfunction and ILD were higher in male than that in female patients in the early stage of SSc.

Disclosure of Interest: None declared

DOI: 10.1136/annrheumdis-2018-eular.1288

\section{AB0811 INCREASED LEVELS OF SERUM WISTERIA FLORIBUNDA AGGLUTININ-POSITIVE MAC-2 BINDING PROTEIN IN SYSTEMIC SCLEROSIS}

Y. Yokoyama ${ }^{1}$, T. Yoshikawa ${ }^{1}$, T. Furukawa ${ }^{1}$, T. Hashimoto ${ }^{1}$, M. Morimoto ${ }^{1}$, N. Azuma ${ }^{1}$, M. Kitano ${ }^{1}$, T. Iwasaki ${ }^{2}$, K. Matsui ${ }^{1}$, H. Sano ${ }^{1} .{ }^{1}$ Division of Rheumatology, Department of Internal Medicine, Hyogo college of Medicine, 1-1 Mukogawa-cho, Nishinomiya-city, Hyogo; ${ }^{2}$ Department of Pharmacy, Hyogo University of Health Sciences, Kobe, Japan

Background: Mac-2 binding protein is a cell-adhesive glycoprotein of the extrac ellular matrix secreted as a ligand of galectin-3 (Mac-2). Recently, a Wisteria floribunda agglutinin positive-M2BP (M2BP) assay developed using a lectin-antibody sandwich immunoassay has shown promise as a new fibrotic marker in liver fibrosis and interstitial lung disease (ILD) to detect unique fibrosis-related glycoalteration. Systemic sclerosis (SSc) is an intractable connective tissue disease that causes skin and organ fibrosis. Progressive fibrosis is now recognised to be one of the major causes of morbidity and mortality in SSc.
Objectives: The aim of this study is to evaluate the utility of serum M2BP levels in patients with SSc.

Methods: We retrospectively measured serum M2BP levels in 76 patients with SSc and 16 healthy controls (HC). We excluded patients of viral hepatitis and cirrhosis. Serum levels of M2BP were measured using $\mathrm{HISCL}^{\circledR}$ M2BP glycosylation isomer Assay Kit. IHC was performed on 5 samples of normal skin, 7 stored samples of SSc skin and 2 samples of SSc-ILD lung tissue. We examined the relationship between serum M2BP levels and clinical parameters in patients with SSc.

Results: The mean age was 62.7 years in SSc patients and 54.6 years in HC. There were 57 limited cutaneous SSc patients and 19 diffuse cutaneous SSc patients. The mean duration of SSc patients was 9.8 years. Serum M2BP levels were significantly higher in patients with SSc than in $\mathrm{HC}(0.853 \pm 0.701$ cutoff index [COI], $0.325 \pm 0.111 \mathrm{COI}$, respectively; $\mathrm{p}<0.00001$ ). Receiver operating characteristic (ROC) curve analysis indicated that serum M2BP correctly differentiated between SSc patients and $\mathrm{HC}$ with a sensitivity of $81.6 \%$ and specificity of $87.5 \%$. In patients with SSc, a significant correlation was not found between serum M2BP levels and age, sex, mRSS, ILD, pulmonary artery hypertension (PAH), SSc types, dermatoscopy findings. In multivariant analysis, disease duration was a only independent factor for higher serum M2BP levels more than $0.64 \mathrm{COI}$, serum M2BP median (odds ratio $0.935,95 \% \mathrm{Cl} 0.878-0.996, \mathrm{p}=0.0385$ ). We detected the protein level of M2BP in SSc patient's fibroblast of skin and SSc Patient's proliferating type II alveolar cells adjacent to fibrotic lesions of lung tissue.

Conclusions: In this study, for the first we demonstrated that M2BP is expressed in skin and lung tissue in SSc patients in IHC and more over the serum M2BP level tends to be higher in patients with short disease duration. It is reveal that M2BP play an important role of fibrosis in early stage of SSc. The serum level of M2BP may be a novel biomarker of SSc.

Disclosure of Interest: None declared

DOI: 10.1136/annrheumdis-2018-eular.5615

\section{AB0812 ULTRASONOGRAPHY FOR THE ASSESSMENT OF SKIN IN SYSTEMIC SCLEROSIS: A SYSTEMATIC REVIEW}

T. Santiago $^{1,2}$, M. Santiago ${ }^{3}$, B. Ruaro ${ }^{4}$, M.J. Salvador ${ }^{1}$, M. Cutolo ${ }^{4}$, J.A. Pereira da Silva ${ }^{1}{ }^{1}$ Rheumatology Department, Centro Hospitalar e Universitário de Coimbra, Portugal; ${ }^{2}$ Faculty of Medicine, University of Coimbra; ${ }^{3}$ Centro Hospitalar e Universitário de Coimbra, Portugal, Coimbra, Portugal; ${ }^{4}$ Research Laboratories and Academic Division of Clinical Rheumatology, Department of Internal Medicine, University of Genova, San Martino Polyclinic, Genova, Italy

Background: Skin involvement is the clinical hallmark of systemic sclerosis (SSc). A palpation-based semi-quantitative tool, the modified Rodnan skin score is the current gold standard for the assessment of skin involvement. However, this method has significant limitations, with emphasis on low sensitivity to change and a high inter-observer variability. Ultrasound has been explored, in the past decades, as a basis for more objective, sensitivity and reproducible measure of skin involvement.

Objectives: To identify and synthesise the best available evidence on the use of ultrasound as a source of skin outcome measures in patients with SSc.

Methods: Two independent reviewers systematically searched Pubmed MEDLINE and Embase (up to December 2017). A study was eligible if it included a least one defined group of patients with SSc, and reported a structured evaluation of the skin with ultrasound and/or ultrasound elastography. This search was aug mented by review of bibliographic references from the included studies. The same two reviewers independently extracted the data and classified the quality of evidence of the included studies by the Effective Public Health Practice Project system.

Results: A total of 30 studies (21 observational cross-sectional and 9 longitudinal) were included. They enrolled a total of 1171 SSc patients, mostly middleaged, female and with a limited form of SSc (59.0\%). The most common ultrasound outcome measure used was skin thickness (in 28 studies), although the definition of this parameter was a highly heterogeneous. Other outcome meas ures were echogenicity (in 7), and/or stiffness (in 6) and/or vascularity (in 1). There was a substantial discordance in the number and exact location of skin sites examined. The main comparator was global and site specific mRSS.

There was a lack of construct and criterion validity (only 1 study assessed the correspondence between ultrasound measures and histological findings). Few stud ies reported information about inter- and intra-rater reproducibility, but when reported, it showed excellent results. Data regarding evidence for responsiveness to change and feasibility were also scarce.

Conclusions: This systematic review highlights a remarkable literature heteroge neity and limited quality of most reported studies. This hinders the evidence cur rently supporting the use of skin ultrasound evaluation in clinical practice, but the very promising data (e.g good reliability and early detection of skin involvement) support its use in clinical research. Further well-designed and dimensioned studies are needed to support the role of skin ultrasound assessment in the early diag nosis and monitoring SSc patients. These may be crucial to improve our 